Reprod. Nutr. Dévelop., 1984, 24 (1), 71-80.

\title{
Variations horaires de la température rectale et de la prolactinémie chez le " Cabrit créole " maintenu à l'extérieur en milieu tropical
}

\author{
P. CHEMINEAU (1), J.-P. RAVAULT $\left({ }^{*}\right)$
}

INRA-CRAAG Station de Zootechnie, 97170 Petit-Bourg, Guadeloupe, France.

(*) Station de Physiologie de la Reproduction, I.N.R.A., Nouzilly, 37380 Monnaie, France.

Summary. Hourly variations in the rectal temperature and plasma prolactin levels of "creole goats " kept outdoors in a tropical environment.

Rectal temperature and plasma prolactin level were measured hourly in " creole goats " during 27 consecutive hours in March, July and November. Simultaneously, air, " black-globe " temperature and relative humidity were measured at the height of the animals.

Rectal temperature and plasma prolactin level varied according to hour and season and were always higher during the day than during the night. Plasma prolactin level increased dramatically for $3 \mathrm{~h}$ after dusk only in March. Rectal temperature showed a significantly higher correlation with air temperature than with "black-globe " temperature. On the contrary, plasma prolactin level was more significantly correlated with «black-globe » temperature than with air temperature. When all seasons and hours were pooled, " blackglobe " temperature showed a threshold $\left(32-34^{\circ} \mathrm{C}\right)$, beyond which prolactin reached values higher than $100 \mathrm{ng} / \mathrm{ml}$. Rectal temperature was always significantly correlated with plasma prolactin level. Plasma prolactin level as an index of thermal stress and the role of prolactin in thermoregulation have been discussed.

\section{Introduction.}

Les animaux des races européennes importées en zone tropicale ont des difficultés d'adaptation à leur nouvel environnement climatique (revue : Ortavant et Loir, 1978). De même, un stress thermique appliqué en chambre climatique entraîne une augmentation rapide de leur température rectale (Sawyer, 1979), de leur prolactinémie (Hooley, Findlay et Stephenson, 1979) et une baisse de leurs performances de reproduction (Sawyer, 1979) ou de croissance (Alexander et Williams, 1971). En revanche peu d'informations sont disponibles sur les races des France.

(1) Adresse actuelle: Station de Physiologie de la Reproduction, Nouzilly, 37380 Monnaie, 
zones tropicales dans leur milieu d'élevage. Sont-elles moins sensibles à leur environnement habituel ? Par quels mécanismes sont-elles adaptées à supporter des températures élevées ? Le " cabrit créole " des Antilles Françaises (Chemineau et al., 1983) a été utilisé dans cette étude pour préciser si cet animal est sensible à son environnement thermique alors qu'il est maintenu à l'extérieur toute la journée " au piquet", mode d'élevage traditionnel dans l'Arc Antillais (Devendra, 1971). En particulier, subit-il des variations de sa température interne, et sa prolactinémie, qui varie dans d'autres espèces avec la charge thermique (bovins : Tucker et Petitclerc, 1982 ; Schams, Stephan et Holley, 1980 ; ovins : Shillo, Alliston et Malvan, 1978), est-elle reliée aux modifications observées de son environnement thermique traditionnel ?

\section{Matériel et méthodes.}

1. Animaux. - Trente femelles multipares de la race locale dite « cabrit créole » sont utilisées en milieu de la phase lutéale induite par l'introduction d'un mâle (Chemineau, 1983). Ces femelles, habituées à être manipulées et à subir des prélèvements sanguins, sont attachées " au piquet " de $7 \mathrm{~h}$ le premier jour de mesure jusqu'à $11 \mathrm{~h}$ le lendemain. Les animaux n'ont pas la possibilité de se mettre à l'ombre et ont à leur disposition le fourrage autour d'eux (Digitaria decumbens stent). Dans le but de reproduire le plus exactement possible les conditions traditionnelles d'élevage, les animaux ont eu la possibilité de s'abreuver à $17 \mathrm{~h}$ le premier jour de mesure en juillet 1979. Aucun des animaux n'ayant voulu boire, la possibilité d'abreuvement a été supprimée aux autres périodes de mesure.

2. Périodes de mesure. - Les mesures ont eu lieu en juillet 1979 (10 femelles), novembre 1979 (10 femelles), mars 1980 (10 femelles) et novembre 1980 (9 parmi les 10 femelles ayant servi en mars 1980). Ces périodes de prélèvement coïncident avec les périodes de ,reproduction du troupeau (Chemineau, 1983), choisies au préalable pour avoir des caractéristiques climatologiques différentes.

3. Prélèvements et enregistrements. - A chacune des périodes de mesure, et de $8 \mathrm{~h}$ le premier jour jusqu'à $10 \mathrm{~h}$ le lendemain, une mesure de la température rectale (thermomètre médical enfoncé de $10 \mathrm{~cm}$ dans le rectum et maintenu en place pendant $1 \mathrm{~min}$ ) et un prélèvement de sang $(5 \mathrm{ml})$ par ponction de la veine jugulaire, sont réalisés toutes les heures sur chaque femelle. Pendant la phase obscure, les prélèvements sont effectués sans lumière artificielle. Chaque prélèvement est placé immédiatement à $+4{ }^{\circ} \mathrm{C}$ puis centrifugé dans les $30 \mathrm{~min}$; le plasma est conservé à - $15^{\circ} \mathrm{C}$ jusqu'au dosage de la prolactine.

Avant chaque prélèvement, la température au centre d'une boule de cuivre (diamètre $12 \mathrm{~cm}$ ) peinte en noir ( = température du " corps noir "), la température de l'air sec et celle de l'air humide (qui permettent le calcul de l'humidité relative) sont enregistrées à proximité immédiate et à hauteur des animaux. La température du corps noir fournit une estimation de la température ressentie par un animal sans sudation. Le début et la fin de la phase obscure sont appréciés «à l'œil " avant le début de chaque prélèvement horaire. 
4. Dosage de la prolactine. - La prolactine caprine ne peut être distinguée immunologiquement de la prolactine ovine et doit donc être estimée en termes de standard ovin (Hart, 1972). Elle est mesurée par la méthode de Kann (1971). Les résultats sont exprimés en ng PRL PS6 $\mathrm{NIH} / \mathrm{ml}$ de plasma. La sensibilité est de $0,3 \mathrm{ng} / \mathrm{ml}$ et les coefficients de variation intra- et inter-dosage sont respectivement de $8 \%$ et $10 \%$.

5. Analyse des résultats. - Les fluctuations de la température rectale et de la prolactinémie rappelant un modèle cyclique de type sinusoïdal la fonction mathématique utilisée est : $y(t)=C o+C \cos (w t+z)$, où t est le temps, Co le niveau moyen des valeurs, $C$ l'amplitude, $z$ la phase et $w$ la fréquence angulaire. La relation entre la période $(\tau)$ et $w$ est la suivante : $\tau=2 \pi / w$. Ces différents paramètres sont estimés par la méthode des moindres carrés non linéaires (algorithme de Gauss-Marquardt : Marquardt, 1963; Halberg et Reinberg, 1967). La validité statistique du rythme est testée selon la méthode décrite par Koukkari et al. (1974).

Le test " $\mathrm{t}$ " et le coefficient de régression linéaire sont également utilisés (Snedecor et Cochran, 1971).

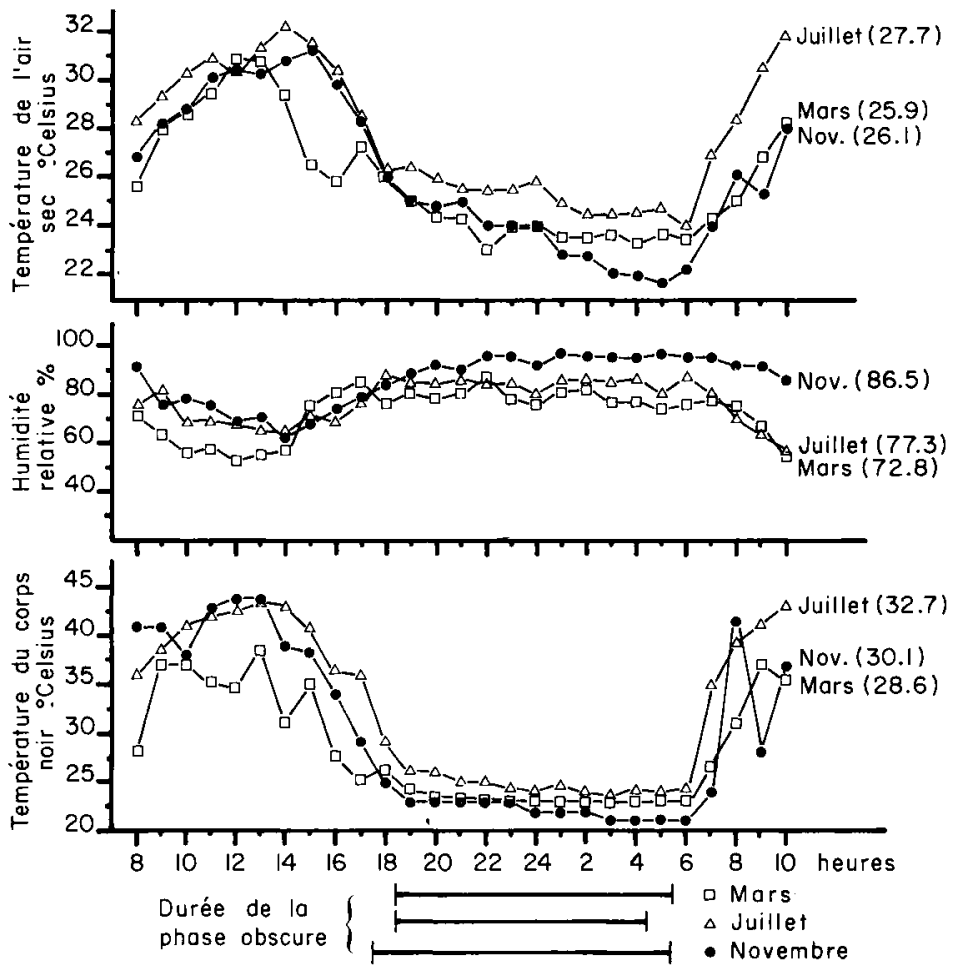

FIG. 1. - Paramètres du climat enregistrés heure par heure au cours des trois premières périodes d'étude $\triangle \longrightarrow \triangle$ juillet, $\triangle \longrightarrow$ novembre 1979, $\square \square \square$ mars; les chiffres entre parenthèses indiquent la moyenne sur la période). 


\section{Résultats.}

1. Paramètres du climat. - Pour une même heure la température de l'air $\mathrm{sec}$, enregistrée au cours des trois premières périodes de mesure, varie peu d'une saison à l'autre $\left(5^{\circ} \mathrm{C}\right.$ maximum). De même la différence entre le jour et la nuit à l'intérieur d'une même saison est faible $\left(9^{\circ} \mathrm{C}\right.$ au maximum en novembre). L'humidité relative est toujours élevée (supérieure à $50 \%$ ) et l'air est proche de la saturation pendant la nuit. L'humidité relative moyenne est plus élevée en novembre qu'en mars et juillet $(p<0,05)$. La température moyenne du corps noir est plus basse en mars qu'aux deux autres saisons $(p<0,05)$ et l'amplitude de la variation jour-nuit $\left(15,5^{\circ} \mathrm{C}\right.$ au minimum en mars) est plus importante que celle de l'air sec. La phase obscure varie de 10 à $12 \mathrm{~h}$ (fig. 1).

En novembre 1980 (même animaux qu'en mars) la température moyenne de l'air sec est plus élevée que celles enregistrées en novembre 1979 et en mars $\left(27,6,26,1\right.$ et $25,9^{\circ} \mathrm{C}$ respectivement; $\left.p<0,05\right)$. La température moyenne du corps noir n'est cependant pas différente de celles enregistrées en novembre 1979 et en mars $\left(29,6,30,1\right.$ et $28,6^{\circ} \mathrm{C}$ respectivement).

2. Température rectale. - Au cours des 3 premières périodes de mesure (fig. 2), les températures rectales les plus élevées sont observées au cours de la journée $(8 \mathrm{~h}$ à $17 \mathrm{~h}$ ) puis, à partir de 17 ou $18 \mathrm{~h}$ selon la saison, elles diminuent progressivement au cours de la nuit pour atteindre les valeurs les plus basses au lever du jour ( $5 \mathrm{~h}$ ou $6 \mathrm{~h}$ ). Ce n'est qu'en juillet qu'un rythme circadien est statistiquement décelable (période $=26 \mathrm{~h} ; \mathrm{F}=26,41, \mathrm{p}<0,001$ ).

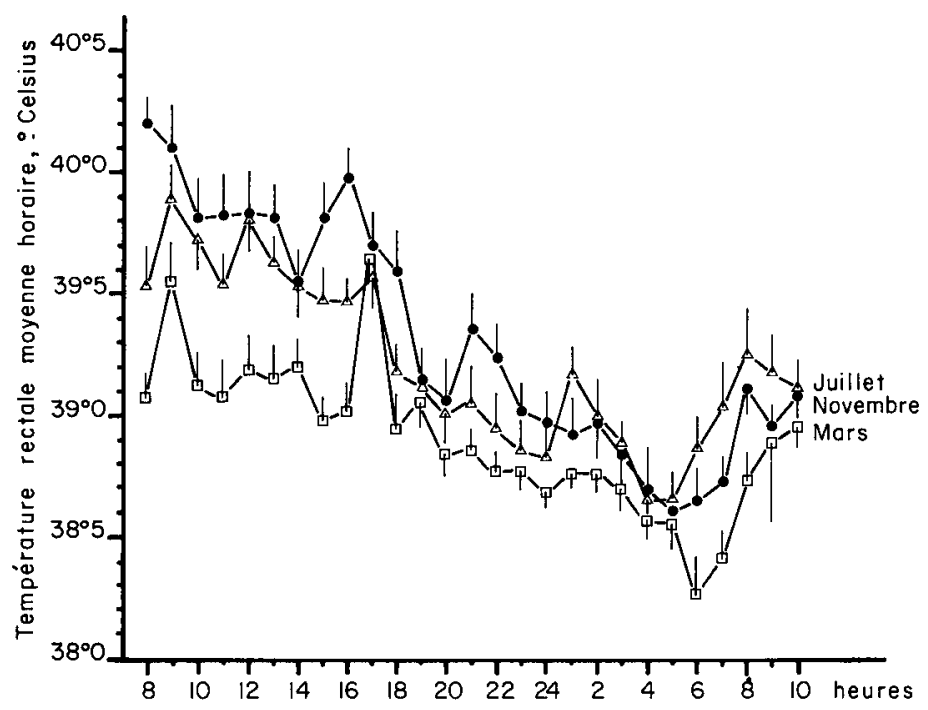

FIG. 2. - Température rectale horaire moyenne $(\mathrm{m} \pm \mathrm{sem})$ enregistrée au cours des trois premières périodes d'étude $\triangle \triangle \triangle$ juillet, $\longrightarrow$ novembre 1979, $\sqcup \longrightarrow \square$ mars). 
Sur l'ensemble du nychtémère, la température rectale moyenne $1 \pm$ erreur standard) des femelles est plus faible en mars qu'en juillet et novembre 1979 $(38,92 \pm 0,08 v s, 39,23 \pm 0,12$ et $39,32 \pm 0,12 ; p<0,05)$ mais n'est pas différente entre juillet et novembre.

3. Prolactine plasmatique. - II existe une variation importante de la prolactinémie horaire moyenne des animaux au cours du nychtémère à chacune des trois premières périodes de mesure (fig. 3) ; toutefois, comme pour la température rectale, c'est uniquement en juillet qu'un rythme est statistiquement mis en évidence (période $=36 \mathrm{~h}, \mathrm{~F}=13,03 ; \mathrm{p}<0,001$ ). Sur l'ensemble du nychtémère, la prolactinémie moyenne $( \pm$ erreur standard) des femelles est plus élevée en juillet qu'en mars $(184 \pm 31 \mathrm{ng} / \mathrm{ml}$ vs $112 \pm 22 \mathrm{ng} / \mathrm{ml} ; \mathrm{p}<0,05)$ et en mars qu'en novembre $1979(112 \pm 22 \mathrm{ng} / \mathrm{ml}$ vs $67 \pm 7 \mathrm{ng} / \mathrm{ml} ; \mathrm{p}<0,10)$. En juillet et novembre 1979, la prolactinémie horaire moyenne des 10 animaux est élevée pendant la période claire et reste faible pendant la période obscure ; en mars, malgré des valeurs diurnes relativement élevées, une brusque augmentation de la teneur plasmatique en prolactine se produit pendant les $3 \mathrm{~h}$ qui suivent la tombée du jour (19, 20 et 21 h) (fig. 3).

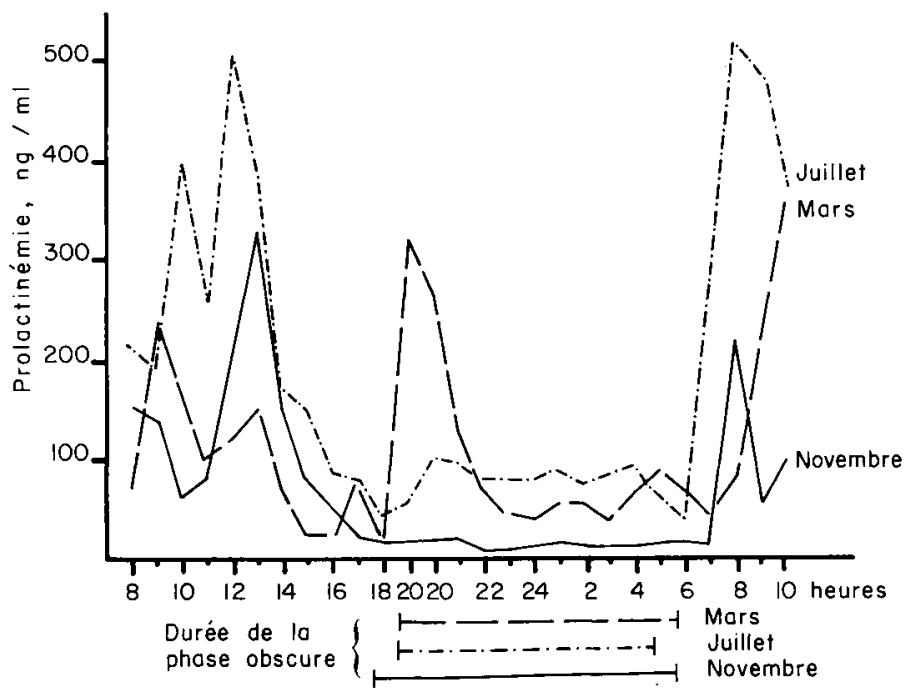

FIG. 3. - Prolactinémie horaire moyenne au cours des trois premières périodes d'étude (-. - juillet, $\longrightarrow$ novembre $1979,-\ldots$ mars).

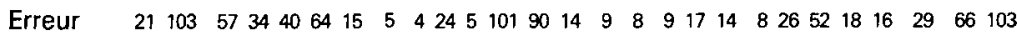
standard de $56 \quad 58 \quad 123 \quad 66 \quad 86 \quad 6922 \quad 3014 \quad 147 \quad 16 \quad 24 \quad 17 \quad 15 \quad 11 \quad 17 \quad 16 \quad 12 \quad 17 \quad 22 \quad 7 \quad 6 \quad 89116 \quad 105 \quad 82$ la moyenne $51 \quad 28 \quad 16 \quad 144577 \begin{array}{lllllllllllllllllllll}33 & 19 & 14 & 3 & 1 & 2 & 9 & 3 & 1 & 2 & 3 & 5 & 3 & 2 & 2 & 4 & 3 & 2 & 36 & 6 & 45\end{array}$

mars juillet novembre

En novembre 1980 (mêmes animaux qu'en mars) la prolactinémie moyenne n'est pas différente de celle observée en novembre $1979(44 \pm 8 \mathrm{ng} / \mathrm{ml}$ vs $67 \pm 7 \mathrm{ng} / \mathrm{ml}$ ) mais elle est beaucoup plus faible que celle de mars (44 $\pm 8 \mathrm{ng} / \mathrm{ml}$ vs $112 \pm 22 \mathrm{ng} / \mathrm{ml} ; \mathrm{p}<0,05$ ) et le pic post-crépusculaire de prolactine observé en mars sur les mêmes animaux, a complètement disparu. 
4. Relations entre paramètres climatiques et physiologiques (tabl. 1, 2 et 3). - Quelle que soit la saison les coefficients de corrélation entre températures du corps noir ou de l'air sec et température rectale ou prolactinémie moyennes horaires sont différents de zéro. Mais, sauf en juillet, la température rectale est mieux corrélée à celle de l'air sec et la prolactinémie mieux à celle du corps noir (tabl. 1 et 2).

\section{TABLEAU 1}

Coefficients de corrélation $(\mathrm{r})$ entre la température rectale moyenne horaire et la température de l'air sec ou celle du corps noir.

\begin{tabular}{|c|c|c|c|c|c|}
\hline & & mars & juillet & novembre 79 & novembre 80 \\
\hline \multirow{2}{*}{$\begin{array}{l}\text { Température } \\
\text { rectale }\end{array}$} & air $\sec \ldots \ldots \ldots \ldots$ & $\begin{array}{c}0,71 \\
* *\end{array}$ & $\begin{array}{l}0,77 \\
\text { NS }\end{array}$ & $\begin{array}{c}0,84 \\
*\end{array}$ & $\underset{*}{0,89(1)}$ \\
\hline & corps noir & 0,51 & 0,79 & 0,77 & $0,82(1)$ \\
\hline
\end{tabular}

Tous les coefficients sont significativement différents de zéro au seuil de $1 \%$; à l'intérieur d'une même saison ces coefficients sont significativement différents entre eux au seuil de ${ }^{*}=5 \%$ ou ** $=1 \%$.

(1) : 9 animaux sur les 10 utilisés en mars.

\section{TABLEAU 2}

Coefficients de corrélation ( $\mathrm{r}$ ) entre la prolactinémie moyenne horaire et la température de l'air sec ou celle du corps noir.

\begin{tabular}{|c|c|c|c|c|c|}
\hline & & mars & juillet & novembre 79 & novembre 80 \\
\hline \multirow{2}{*}{$\begin{array}{c}\text { Prolactine } \\
\text { plasmatique }\end{array}$} & $\cdots \cdots \cdots \cdots$ & $0,50\left(^{(1)}\right.$ & 0,65 & $\underset{* *}{0,64}$ & $0,75(2)$ \\
\hline & corps noir . ....... & $0,67(1)$ & 0,77 & 0,84 & $0,82(2)$ \\
\hline
\end{tabular}

Tous les coefficients sont significativement différents de zéro au seuil de $1 \%$; à l'intérieur d'une même saison ces coefficients sont significativement différents entre eux au seuil de ${ }^{*}=5 \%$ ou ${ }^{* *}=1 \%$.

(1) : Après exclusion des trois prélèvements qui suivent le crépuscule.

(2) : 9 animaux sur 10 utilisés en mars.

\section{TABLEAU 3}

Coefficients de corrélation $(\mathrm{r})$ entre température rectale et prolactinémie moyennes horaires.

\begin{tabular}{|c|c|c|c|}
\hline $\begin{array}{l}\text { mars } \\
0,35^{*}\end{array}$ & $\begin{array}{c}\text { juillet } \\
0,49^{* *}\end{array}$ & $\begin{array}{c}\text { novembre } 79 \\
0,53^{* * *}\end{array}$ & $\begin{array}{c}\text { novembre } 80 \\
0,61^{* * *}\end{array}$ \\
\hline
\end{tabular}


Toutes saisons confondues (fig. 4) il est possible de distinguer un seuil de température du corps noir $\left(32-34^{\circ} \mathrm{C}\right)$ au-delà duquel la prolactinémie peut prendre des valeurs supérieures à $100 \mathrm{ng} / \mathrm{ml}$.

La température rectale moyenne horaire est, à chaque saison, corrélée avec la prolactinémie moyenne horaire (tabl. 3 ).

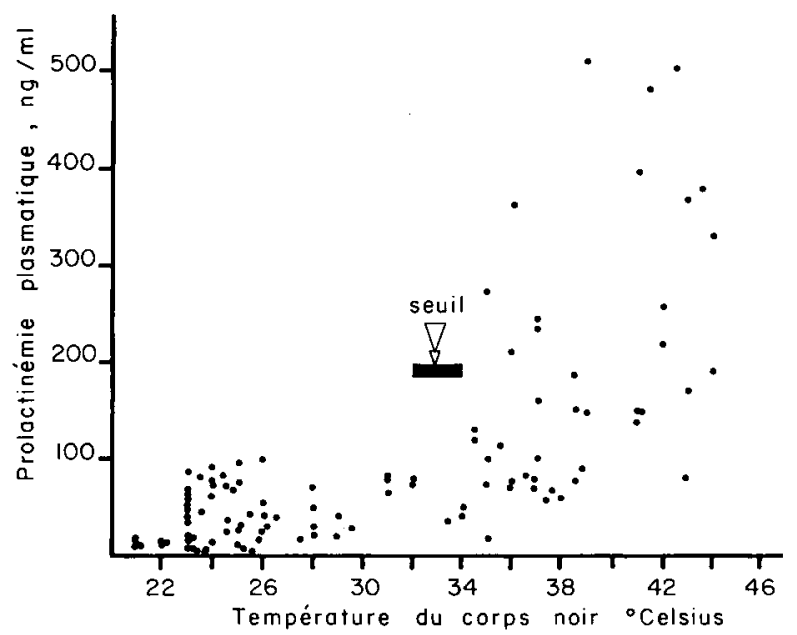

FIG. 4. - Prolactinemie plasmatique horaire movenne et température du corps noir (toutes saisons confondues; points de 19-20-21 h de mars exlus).

\section{Discussion.}

L'enregistrement de la température rectale d'animaux maintenus dans les conditions traditionnelles d'élevage " au piquet " montre qu'il existe une variation de la température interne des animaux liée à la saison et à l'heure du nychtémère. La variation saisonnière se manifeste surtout pendant la période claire ; aux deux saisons les plus chaudes, les animaux augmentent leur température interne pendant la journée. Quelle que soit la saison celle-ci diminue progressivement pendant la nuit pour atteindre son minimum en fin de nuit. II est toutefois difficile de mettre en évidence statistiquement un rythme circadien, probablement parce que la température rectale est reliée étroitement aux paramètres climatiques (température de l'air et du corps noir). Ceci indique que la chèvre créole est sensible à son environnement thermique puisque l'on explique une part importante $(r>0,50)$ de la variance de la température rectale avec l'une ou l'autre de ces deux données climatiques. Aux saisons les plus chaudes (juillet et novembre) les femelles semblent incapables d'empêcher une élévation de leur température interne.

Peut-être faut-il rechercher cette incapacité dans la faible taille des animaux dont la " capacité de stockage thermique " serait insuffisante pour éviter cette augmentation de température. Toutefois en l'absence de données sur les réactions de lutte contre la chaleur (sudation, polypnée) et de données en ambiance constante, il est difficile de préciser si ces variations sont dues : 
$1^{\circ}$ à des variations subies lorsque les défenses contre le chaud sont débordées (hyperthermie vraie);

$2^{\circ}$ à une variation spontanée de la température réglée (indépendante de la température ambiante) ;

$3^{\circ}$ ou bien encore à des variations accompagnant la température ambiante qui sont réglées par l'organisme pour diminuer l'effort de lutte contre le chaud. Le cabrit créole réagit alors, dans ce dernier cas, de la même façon que le dromadaire dont la température rectale varie de $6^{\circ} \mathrm{C}$ entre le jour et la nuit (SchmidtNielsen et al., 1957).

La mesure de la prolactinémie horaire indique clairement que la sécrétion de prolactine est plus élevée pendant lá journée que pendant la nuit au contraire de ce qui est observé généralement quelle que soit la photopériode, dans des conditions tempérées (bélier : Ravault et al., 1980 ; brebis : Cognié, Oldham, Gayerie et Ravault, résultats non publiés; taureau : Schams et al., 1978). II n'y a qu'en mars, saison la moins chaude, que le pic habituel qui suit le crépuscule (bélier: Ravault et al., 1980 ; brebis : Cognié, Oldham, Gayerie et Ravault, résultats non publiés) est observé. Ce pic post-crépusculaire est bien lié à la saison d'observation et non à des différences individuelles puisque, sur les mêmes animaux, il n'apparaît plus du tout en novembre 1980. Comme pour la température rectale, la difficulté de mise en évidence d'un rythme circadien, montre que la prolactinémie est étroitement reliée aux paramètres du climat. En effet les mécanismes impliqués dans la lutte contre la chaleur mettent en jeu la sécrétion de prolactine puisqu'il existe une forte liaison entre le niveau plasmatique de cette hormone et les paramètres thermiques du climat. Les meilleures corrélations obtenues entre prolactinémie et température du corps noir qu'entre prolactinémie et température de l'air indiquent que les modifications diurnes du rayonnement solaire (dues à l'occultation du soleil) sont répercutées immédiatement sur la prolactinémie au contraire de ce qui est observé sur la température rectale. La mise en évidence d'un seuil de température du corps noir, au-delà duquel se déclenche l'hypersécrétion prolactinique, laisse croire que c'est à partir de celui-ci que sont mis en jeu les mécanismes régulateurs de la température interne.

Au-delà de ce seuil la prolactine pourrait alors participer à des modifications des métabolismes hydrique et minéral (Burstyn, 1978) qui permettraient à l'animal de s'adapter à des conditions difficiles et expliqueraient le refus d'abreuvement observé lors de la première période d'étude (Robertshaw, 1982). Le taux plasmatique de prolactine constituerait un bon indicateur du déclenchement de ces mécanismes. Chez les bovins, le blocage de cette hypersécrétion prolactinique qui suit la charge thermique n'entraîne pas de modification des réactions de lutte contre la chaleur (rythme respiratoire, température rectale) mais induit des différences dans l'excrétion urinaire du potassium et du sodium (Schams, Stephan et Holley, 1980). Chez tous les vertébrés, y compris les Mammifères, la prolactine semble être impliquée dans de nombreux phénomènes d'osmorégulation (Loretz et Bern, 1982). Dans d'autres espèces le stress thermique entraîne rapidement une hyperprolactinémie (brebis : Hooley, Findlay et Stephenson, 1979; Schillo, Alliston et Malven, 1978 ; vache : Wettemann et Tucker, 1974 ; Tucker et Petitclerc, 
1982) ; mais dans ces expériences les animaux sont maintenus en chambres climatiques et non pas dans leur système traditionnel d'élevage. Les taux élevés de prolactine observés ici en juillet, pourraient également être à V'origine des perturbations de la fonction ovarienne (cycles courts) observés sur fa même race et à la même saison (Chemineau et al., 1983). Les corrélations significatives obtenues entre la température rectale et la prolactinémie montrent que la prolactine est bien impliquée dans la régulation de la température interne des animaux. II est toutefois difficile de préciser si la sécrétion de la prolactine accompagne la mise en place du mécanisme de régulation thermique ou fait partie de celui-ci. D'autres hormones proches de la prolactine sont en effet également mises en jeu dans la régulation de la température interne (mélatonine: Lynch, Sullivan et Gendler, 1980) ou la réponse au stress thermique (thyroïdiennes: Chauduri et Sadhu, 1961 ; Yousef, Kibler et Johnson, 1967). L'étude réalisée ici ne permet cependant pas de savoir si cette hyperprolactinémie induite par l'environnement thermique des animaux a des répercussions sur leurs performances de reproduction ou de croissance.

Reçu en juillet 1982.

Accepté en octobre 1983.

Remerciements. - Les auteurs tiennent à remercier le NIH pour la fourniture de prolactine, Paul Berbigier (Station de Bioclimatologie, INRA, Petit-Bourg) pour ses conseils, Yves Cognié et Nati Poulin (INRA, Nouzilly) pour leur aide et Marie-Françoise Pinault pour la dactylographie de ce texte.

\section{Références}

ALEXANDER G., WILLIAMS D., 1971. Heat stress and development of the conceptus in domestic sheep. J. agric. Sci., Camb., 76, 53-72.

BURSTYN P. G. R., 1978. Sodium and water metabolism under the influence of prolactin, aldosterone and anti-diuretic hormone. J. Physiol., 275, 39-50.

CHAUDHURI S., SADHU D. P., 1961. Thyroid activity at higher ambiant temperatures. Nature, 192, 560-561.

CHEMINEAU P., 1983. The effect on oestrus and ovulation of exposing creole goats to the male at three times of the year. J. Reprod. Fert., 67, 65-72.

CHEMINEAU P., COGNIÉ Y., XANDE A., PEROUX F., ALEXANDRE G., LeVY F., SHITALOU E., BECHE J. M., SERGENT D., CAMUS E., BARRE N., THIMONIER J., 1983. Le cabrit créole et ses caractéristiques zootechniques. Rev. Elev. Méd. vét. Pays trop. laccepté pour publication).

DEVENDRA C., 1971. Goat production in the carribbean. Ind conf. int. Elevage Caprin, Tours, 47-54.

HALBERG F., REINBERG A., 1967. Rythmes circadiens et rythmes de basses fréquences en physiologie humaine. J. Physiol., 59, 117-200.

HART I. C., 1972. A solid phase radioimmunoassay for ovine and caprine prolactin using sepharose $6 \mathrm{~B}$ : its application to the measurement of circulating levels of prolactin before and during parturition in the goat. J. Endocrinol., 55, 51-62.

HOOLEY R. D., FINDLAY J. K., STEPHENSON R. G. A., 1979. Effect of heat stress on plasma concentrations of prolactin and luteinizing hormone in ewes. Aust. J. biol. Sci, 32, 231-235.

KANN G., 1971. Dosage radioimmunologique de la prolactine plasmatique chez les ovins. $C$. $R$. Acad. Sci. Paris, Sér. D, 272, 1808-1811. 
KOUKKARI W., DUKE S. H., HALBERG F., LEE J. K., 1974. Circadian rhythmic leaflet movements : student exercise in chronobiology. Chronobiologia, 1, 281-302.

LORETZ C. A., BERN H. A., 1982. Prolactin and osmoregulation in vertebrates. An update. Neuroendocrinology, 35, 292-304.

LYNCH G. R., SULLIVAN J. K., GENDLER S. L., 1980. Temperature regulation in the mouse, Peromyscus leucopus : effects of various photoperiods, pinealectomy and melatonin administration. Int. J. Biometeor., 24, 49-55.

MAROUARDT D. W., 1963. An algorithm for least-squares estimation of non linear parameters. J. Soc. indust. appl. Math., 11, 431-441.

ORTAVANT R., LOIR M., 1978. The environment as factor in reproduction in farm animals. 4 e World Congr. anim. Prod., 20-26 April 1978, Buenos Aires, Vol. 1, 423-451.

RAVAULT J. P., BLANC M., ORTAVANT R., PELLETIER J., de REVIERS M. M., 1980. Variations circadiennes et circanuelles de la sécrétion de prolactine (Prl), LH et FSH chez les animaux domestiques mâles, 125-128. In R. ORTAVANT, A. REINBERG, Rythmes et reproduction. Masson et Cie, Paris.

ROBERTSHAW D., 1982. Concepts in animal adaptation (thermoregulation of the goat), 395-397. In Proc. 3rd int. Conf. on goat production and disease. January 10-15 1982.

SAWYER G. J., 1979. The influence of radiant heat load on reproduction in the Merino ewe. Aust. J. agric. Res., 30, 1133-1162.

SCHAMS D., GOMBE S., SCHALLENBERGER E., REINHARDT V., CLAUS R., 1978. Relationships between short term variations of $\mathrm{LH}, \mathrm{FSH}$, prolactin and testosterone in peripheral plasma of prepubertal bulls. J. Reprod. Fert., 54, 145-148.

SCHAMS D., STEPHAN E., HOOLEY R. D., 1980. Effect of prolactin inhibition under heat exposure on water intake and excretion of urine, sodium and potassium in bulls. Acta endocr., 94, 315320.

SCHILLO C., ALLISTON C. W., MALVEN P. V., 1978. Plasma concentrations of luteinizing hormone and prolactin in the ovariectomized ewe during induced hyperthermia. Biol. Reprod., 19, 306-313.

SCHMIDT-NIELSEN K., SCHMIDT-NIELSEN B., JARNUM S. A., HOUPT T. R., 1957. Body temperature of the camel and its relation to water economy. Am. J. Physiol., 188, 103-112.

SNEDECOR G. W., COCHRAN W. G., 1971. Statistical methods. 6th ed. The lowa Univ. Press.

TUCKER H. A., PETITCLERC D., 1982. The role of the eye on secretion of prolactin during various photoperiods and seasons in cattle. In photoperiodism and reproduction in vertebrates, 147 . 156. In R. ORTAVANT, J. PELLETIER, J. P. RAVAULT, Coll. INRA, $\mathrm{n}^{\circ} 6$.

WETTEMANN R. P., TUCKER H. A., 1974. Relationship of ambient temperature to serum prolactin in heifers. proc. Soc. exp. Biol. Med., 146, 908-911.

YOUSEF M. K., KIBLER H. H., JOHNSON H. D., 1967. Thyroid activity and heat production in cattle following sudden ambient temperature changes $J$. anim. Sci., 26, 142-148. 